\title{
Data Monitoring
}

National Cancer Institute

\section{Source}

National Cancer Institute. Data Monitoring. NCI Thesaurus. Code C142488.

Review of clinical information to determine veracity, thoroughness, and dependability. 